\title{
Thermally assisted oscillatory interlayer exchange bias coupling
}

\author{
Minn-Tsong Lin, ${ }^{1, *}$ C. H. Ho, ${ }^{1}$ Ching-Ray Chang, ${ }^{1}$ and Y. D. Yao ${ }^{2}$ \\ ${ }^{1}$ Department of Physics, National Taiwan University, 106 Taipei, Taiwan \\ ${ }^{2}$ Institute of Physics, Academia Sinica, 115 Taipei, Taiwan
}

(Received 22 December 2000; published 9 February 2001)

\begin{abstract}
A strong temperature dependence of the characteristic behavior of the interlayer exchange bias coupling was observed in a ferromagnet/nonmagnetic metal/antiferromagnet trilayer system $(\mathrm{NiO} / \mathrm{Cu} / \mathrm{NiFe})$. The oscillation of the interlayer exchange bias coupling was found to be thermally assisted. At low temperature, the exchange bias field decreased monotonically with the $\mathrm{Cu}$ spacer thickness. Increasing the temperature close to the Neél temperature, the interlayer exchange bias field became oscillatory with the $\mathrm{Cu}$ spacer thickness. A simple picture of the temperature-dependent competition between the RKKY-like coupling and the antiferromagnetic coupling within the antiferromagnetic layer as well as the interlayer dipolar interaction is proposed to explain these findings.
\end{abstract}

DOI: 10.1103/PhysRevB.63.100404

PACS number(s): 75.70.Cn

The discovery of the long-range magnetic coupling between magnetic layers across a conductive spacer is one of the most important advances in low-dimensional magnetic systems in recent years. At variation of spacer thickness, the interlayer coupling between two ferromagnetic layers behaves oscillatory and alternates between ferromagnetic (FM) and antiferromagnetic (AF) coupling. ${ }^{1,2}$ The RKKY-like models have been successfully used to explain this oscillatory behavior of the interlayer coupling by considering the interference of the conduction electrons of one magnetic layer with those in another magnetic layer across the Fermi surface of the spacer. ${ }^{3,4} \mathrm{Up}$ to now, this kind of the interlayer coupling has been intensively studied in experiment as well as in theory and is basically well understood under RKKYlike picture.

On the other hand, the physics of the magnetic coupling between AF and FM layers is more complicated as compared to the former one. The direct coupling between AF and FM layers in an FM/AF bilayer system reveals a so-called exchange bias coupling, which results in an additional unidirectional anisotropy, leading to a shift of the magnetic hysteresis loops. The physical origin of the exchange bias coupling was firstly assumed to be a kind of nearest-neighbor or short-range exchange coupling at interface. ${ }^{5,6}$ Most previous studies indicated a strong dependence of the exchange bias field on the interface spin structure. These are, for example, a spin-flop interface state ${ }^{7}$ as well as existence of a domain wall ${ }^{8}$ for positive exchange bias field in $\mathrm{FeF}_{2} / \mathrm{Fe},{ }^{9}$ effect of interface roughness, ${ }^{9,10}$ the relative orientation of the FM and AF spin at interface, ${ }^{10}$ and effects of the temperature and cooling field on the surface spin structure of the AF layer. ${ }^{11}$

A pioneer experimental study has, however, indicated that the exchange bias field is a kind of long-range interaction, which may extend, in a way of exponential decay, several tens of $\AA$, depending on the spacer material or most likely specific electronic structure in nature. ${ }^{12}$ Although this finding indicated that the FM layer may correlate with the AF layer via the conduction electrons across the nonmagnetic conductive layer, an oscillatory behavior of the exchange bias field, as expected in an RKKY-like model for the FM/NM/FM system, was, however, not found in this work.

Very recent works, in contrast to the finding in Ref. 12, reported nonmonotonic variation of the exchange bias field with the nonmagnetic spacer thickness. ${ }^{13,14}$ The nonmonotonic behavior found in Ref. 13 was attributed to the interface roughness. The one observed in Ref. 14 revealed an oscillatory evolution, however, superposed with a strong monotonic background, and had a monolayer oscillation period. The effect due to the interface roughness, however, can not yet be completely excluded. The contradictory results in Ref. 14 and Ref. 12 and the physical origins which determines the characteristic behavior of the interlayer exchange bias coupling still needs to be clarified.

In this communication, we present a thermally assisted oscillatory nature of the long-range exchange bias coupling across nonmagnetic spacer $\mathrm{Cu}$ at variation of thickness. The oscillatory behavior of the interlayer exchange bias coupling is strongly depressed at low temperature, and the exchange bias field became monotonic decaying with the spacer thickness. The finding of the significant temperature dependence clarifies the contradiction in the previous studies. ${ }^{12,14}$ It also indicates a temperature-dependent competition mechanism, in which the interlayer RKKY-like coupling competes with the effective AF coupling within the AF layers and the interlayer dipolar interaction, and dominates the behavior of the interlayer exchange bias field at temperatures close to the Neél temperature $T_{N}$ of the AF layer.

The magnetron sputtering system with a base pressure lower than $3 \times 10^{-7}$ torr was used in 2 mtorr Ar working pressure for deposition of the FM layers (100 $\AA \mathrm{NiFe}), \mathrm{Cu}$ layers and $\mathrm{AF}(100$ and $250 \AA \mathrm{NiO})$ layers on the $\mathrm{Si}(110)$ substrate, using DC and RF power sources for conductive and nonconductive layers, respectively. The thickness of the layers was carefully calibrated by quartz thickness monitor and Detak surface texture probing system, and was, in particular for the $\mathrm{Cu}$ spacer, well controlled within $10 \%$ deviation of the desired value for each deposition. The thicknesses of the $\mathrm{NiO}$ layers were intentionally chosen to be 100 and $250 \AA$, which have different $T_{N}(\sim 200$ and $\sim 350 \mathrm{~K}$ estimated from the blocking temperatures), due to the finite-size effect. $^{15}$ 


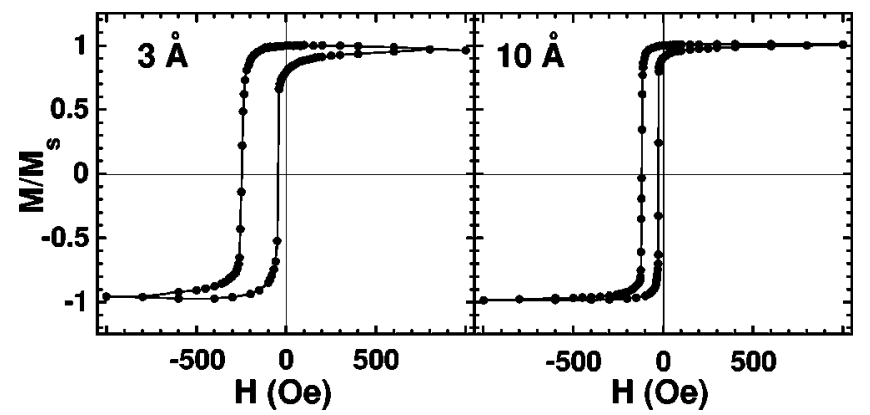

FIG. 1. Hysteresis loops taken at $20 \mathrm{~K}$ for the $100 \AA \mathrm{NiO} / \mathrm{X}$ $\mathrm{Cu} / 100 \AA \mathrm{NiFe}$ with $x=3$ and $10 \AA$ after the 1000 Oe field cooling procedure.

The magnetic hysteresis loops were obtained by a superconducting quantum interference device. Before magnetic measurements, the sample was first cooled under an external field 1000 Oe from $300 \mathrm{~K}$ (or $360 \mathrm{~K}$ ), which is above the $T_{N}$ of the $100 \AA$ (or $250 \AA$ ) NiO, down to the desired measurement temperature. The 1000 Oe cooling field is high enough to form a well ordering of the AF layer.

Figure 1 shows the representative examples of the hysteresis loops taken at $20 \mathrm{~K}$ for the $100 \AA \mathrm{NiO} / \mathrm{X} \mathrm{Cu} / 100 \AA \mathrm{NiFe}$ with Cu spacer thickness $x=3 \AA$, and $10 \AA$ after the 1000 Oe field cooling procedure. A significant shift in all hysteresis loops from the $H=0$ is observed.

In Fig. 2(a), the values of the $H_{e}$ at different temperatures of $100 \AA \mathrm{NiO} / \mathrm{Cu} / 100 \AA \mathrm{NiFe}$ are depicted as a function of the spacer thickness. The $H_{e}$ measured at $20 \mathrm{~K}$ behaves as a long-range interaction, decreasing monotonically with increasing Cu thickness, and vanishes above $25 \AA$. This finding agrees well with the previous finding in $\mathrm{Py} / \mathrm{Cu} / \mathrm{CoO}$ systems. ${ }^{12}$ The characteristic behavior of the evolution of the $H_{e}$ with the spacer thickness changes, however, significantly when the temperature varies. It should be noted that each measurement at a certain temperature possesses its own field cooling procedure, and is independent of the measurements at other temperatures. Starting with the temperature $70 \mathrm{~K}$, the $H_{e}$ becomes oscillatory with $\mathrm{Cu}$ thickness. This tendency increases with increasing temperature. At $145 \mathrm{~K}$, which is close to the $T_{N}$, an evident oscillation, without any background subtraction, of the $H_{e}$ value is observed for the thickness range between 3 and $14 \AA$, indicating an oscillatory interlayer bias coupling. The period of the oscillation counts for about $11 \AA$, which is consistent with the long period in the interlayer exchange coupling in $\mathrm{Co} / \mathrm{Cu} / \mathrm{Co}$ as well as $\mathrm{Fe} /$ $\mathrm{Cu} / \mathrm{Fe}$ (Refs. 16 and 17) and in the magnetoresistance oscillation for $\mathrm{Co} / \mathrm{Cu}(100)$ multilayers. ${ }^{18}$ This indicates that the characteristic behavior of the oscillation for both FM/ NM/FM and FM/NM/AF systems can be traced back to the same origin in electronic structure specified by the spacer materials. For $\mathrm{Cu}$ thickness larger than $14 \AA$, the $H_{e}$ of the $\mathrm{NiO} / \mathrm{Cu} / \mathrm{NiFe}$ becomes too small to observe any characteristic behavior. Increasing the temperature up to $200 \mathrm{~K}$, the $H_{e}$ vanishes for all $\mathrm{Cu}$ thickness, reaching the $T_{N}$ of the $\mathrm{AF}$ layer.

Different from the half value of the short exchange coupling period found in Ref. 14, the period observed here is, as

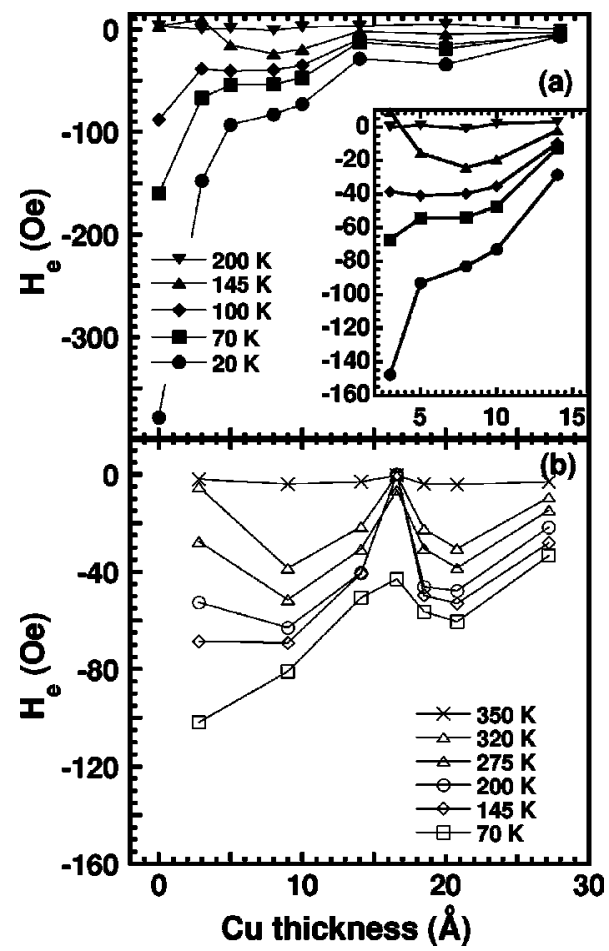

FIG. 2. (a) The $H_{e}$ values for $100 \AA \mathrm{NiO} / \mathrm{Cu} / 100 \AA \mathrm{NiFe}$ as a function of the $\mathrm{Cu}$ thickness at various temperatures of 20, 70, 100, 145 , and $200 \mathrm{~K}$. The inset includes the same data, however, in a different scale for a clear presentation of their characteristic behavior. (b) The same as in (a), but for $250 \AA \mathrm{NiO} / \mathrm{Cu} / 100 \AA \mathrm{NiFe}$ at 70 , $145,200,275,320$, and $350 \mathrm{~K}$. Note that the significant oscillatory behavior appears at higher temperature for $250 \AA \mathrm{NiO}$ due to the higher $T_{N}(\sim 350 \mathrm{~K})$ as compared to $100 \AA \mathrm{NiO}\left(T_{N} \sim 200 \mathrm{~K}\right)$.

indicated above, consistent with that in FM/NM/FM system. ${ }^{16-18}$ In our case, the in-phase and out-of-phase interference between FM and AF layers, which are corresponding to the FM and AF coupling in FM/NM/FM, respectively, must give the different (oppose) contribution to the $H_{e}$. It is, however, hardly to explain the half periodicity (1 ML) or the $|\cos (2 \pi d / \lambda)|$ dependence of the $H_{e}$ as reported in Ref. 14. As will be discussed below, a temperature-dependent measurement could be helpful for checking the possible effect of the interface roughness on the $H_{e}$, which may also oscillate with film growing in a period of monolayer.

For the samples with $250 \AA \mathrm{NiO}$ layer, as shown in Fig. 2(b), the $H_{e}$ reveals a similar characteristic behavior. The oscillation period $(11 \AA)$ is the same as that with $100 \AA \mathrm{NiO}$. It should be noted that the temperature range, in which the oscillation appears, is shifted to higher temperature. This is due to the higher $T_{N}$ for the $250 \AA \mathrm{NiO}$ layer as mentioned above. It is clear to see for both $\mathrm{NiO}$ thicknesses, that at low temperatures, the oscillatory behavior is suppressed. Temperature here plays a crucial role for the interlayer exchange bias coupling. The oscillatory behavior is thermally assisted, and more significantly, emerges only within the temperature range close to the $T_{N}$.

The finding that the $H_{e}$ oscillation is thermally assisted excludes also the doubt if the (extrinsic) effects of the interdiffusion and interface roughness cause the oscillatory be- 


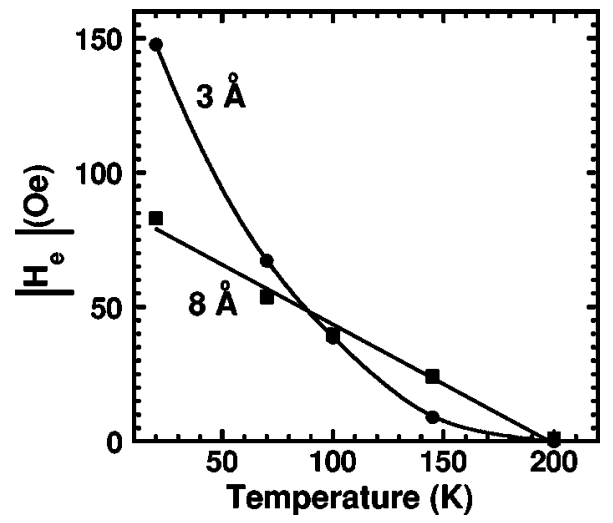

FIG. 3. The $H_{e}$ values of the samples with 3 and $8 \AA \mathrm{Cu}$ spacer as a function of temperature. The data for $8 \AA$ is fitted linearly. The solid line for $3 \AA$ serves as an eye guide only.

havior. Since the interface morphology should be unchanged within our measurement temperature range, the oscillation should be found at all temperature investigated if it is really induced by the roughness or interdiffusion. Our finding is clearly not the case, and should be due to the intrinsic properties as mentioned above. In fact, the exchange bias can be really enhanced in a diluted antiferromagnets, however, only with its volume part, but not interface. ${ }^{19}$

In order to pick up the temperature-dependence feature, the $H_{e}$ of the samples with 3 and $8 \AA$ spacer, which are corresponding to the minimal and maximal points of the oscillation in Fig. 2(a), respectively, is depicted as a function of temperature in Fig. 3. It is easy to see that they reveal significantly different temperature dependence and have a cross point at $\sim 85 \mathrm{~K}$. The data for the $8 \AA$ can be fitted well linearly, while being more complicated for the $3 \AA$.

As mentioned above, the behavior of the exchange bias field is often traced back to the spin configuration or microstructure at interface, which may be temperature dependent. $^{11}$ Nevertheless, different from the AF/FM bilayer, the spin configuration at interface of the FM/NM/AF trilayer is not only determined by the AF coupling or intrinsic spin structure within AF layers, but also affected by the long-range interlayer interaction across the spacer with the FM layer. The characteristic behavior of the exchange bias field should be thus attributed to both AF and interlayer couplings.

To obtain a clear picture for understanding our findings, we consider an AF/NM/FM trilayer system with a constant FM thickness and magnetization, in which the anisotropic effect is negligible, and the FM layer magnetically homogeneous. We may express the energy $E_{b}$ of the exchange bias system in a simple model as follows,

$$
E_{b}=J_{\text {inter }}(T, d) S_{F M} S_{A F, i}+J_{A F}(T) S_{A F, i} S_{A F},
$$

where $S_{F M}$ is the spin of the FM layer, $S_{A F, i}$ the effective uncompensated spin at interface, which participates in the exchange bias interaction with the FM layer, $S_{A F}$ the neighbor spin within the AF layer, $J_{\text {inter }}$ the effective interlayer coupling between the FM and AF interface layers (i.e., $S_{F M}$ and $\left.S_{A F, i}\right), J_{A F}$ the effective antiferromagnetic coupling be- tween $S_{A F, i}$ and $S_{A F} \cdot J_{\text {inter }}(T, d)$, a long-range coupling, depends on the temperature and the spacer thickness $d$. The effective AF coupling $J_{A F}(T)$ includes the temperature dependence due to the thermal fluctuation of the surface spin in the usual form, such as the Brillouin function in the classical ferromagnet. For the $d=0 \AA$, the $J_{\text {inter }}(T, 0)$ becomes $J_{A F-F M}(T)$ (the interface coupling between AF and FM spins), while the $J_{A F}(T)$ is the AF coupling between two AF spins, as suggested in Koon's model.

The long-range interlayer coupling $J_{\text {inter }}$ may have two different physical origins. The first one is the RKKY-like interlayer coupling mentioned above. The second possible source of the interlayer coupling is the dipolar interaction or magnetostatic coupling $J_{\text {dip }}$, which critically depends the net AF surface spin due to presence of the surface or interface roughness. The temperature dependence of $J_{\text {dip }}$ is attributed to the thermal fluctuation of the magnetic moments of the AF layers at interface and FM layers. The presence of the roughness at interface gives a nonzero contribution to the coupling, which may be comparable to the RKKY one $\left(J_{R K K Y}\right) \cdot{ }^{20-23}$ A detailed estimation on the strength of dipolar interaction needs however further information on the interface roughness.

The $T$ dependence of $J_{R K K Y}$ is, however, more complicated, and has been attributed to two different origins. ${ }^{24}$ The first one derives from the disordering of the spin moments. It has been shown that, for thicker FM layers of the FM/ NM/FM system, the RKKY-coupling decays almost linearly and slowly with $T$ near the $T_{C},{ }^{25}$ while with $T \ln T$ for a monolayer limit. ${ }^{26}$ The second one is caused by the intrinsic $T$ dependence of the electronic structure of the layers due to smoothing out the step feature of the Fermi distribution at finite temperatures. ${ }^{24,27}$ Since the $d$ determines the oscillation amplitude of the periodic term in the $J_{R K K Y}$, the $J_{R K K Y}$ at various $d$ gives, therefore, different contribution to the $E_{b}$, leading to the different $T$ dependence of the interlayer exchange bias coupling, as shown above in Fig. 3. This makes also the $T$ dependence of the interlayer exchange bias coupling more complicated as compared to the interlayer coupling in FM/NM/FM systems, and still requires further theoretical input for the quantitative understanding.

To explain our findings qualitatively, we may now propose a simple picture, in which the $J_{R K K Y}$ competes temperature dependently with $J_{d i p}$ and $J_{A F}$. The $J_{R K K Y}$ decays slowly with the temperature. At high temperatures close to the $T_{N}$, it may overcome both $J_{\text {dip }}$ and $J_{A F}$ couplings, and therefore the $H_{e}$ become oscillatory with the spacer thickness, as found above for both series of samples.

On the other hand, at low temperatures, the $J_{A F}$ and $J_{d i p}$ are dominant, and the oscillatory behavior due to the $J_{R K K Y}$ is suppressed. The mechanism of the competition between $J_{R K K Y}$ and $J_{d i p}$ as well as $J_{A F}$ determines thus the characteristic behavior of the interlayer exchange bias field $H_{e}$. This also clarifies the contradiction in the previous studies, ${ }^{12,14}$ and explains the absence of the $H_{e}$ oscillation for the Py/ spacer/CoO sample in the previous work, ${ }^{12}$ in which the measurement temperature $(80 \mathrm{~K})$ could be too low with respect to the $T_{N}(290 \mathrm{~K})$ of the $\mathrm{CoO} \mathrm{AF}$ layer. 
Finally, besides the simple picture above, one seems not to be able to completely exclude the possibility that the interface could somehow affect the temperature-dependent behavior of the interlayer coupling through the probable presence of the pinhole. The NiFe in the pinholes could be highly magnetic at low temperature and the direct exchange bias mechanism may thus overcome the RKKY coupling, while at high temperature, the RKKY-coupling dominates because $\mathrm{NiFe}$ in the pinhole is only weakly magnetic. Nevertheless, as indicated above in Fig. 2, the temperature range associated with apparent $H_{e}$ oscillation for the trilayers with $250 \AA \mathrm{NiO}$ is much higher than that with $100 \AA \mathrm{NiO}$. Since the thickness of the FM layer and in turn its $T_{C}$ was kept unchanged, the enhanced temperature range for the $H_{e}$ oscillation found in Fig. 2(b) is thus most likely attributed to the higher $T_{N}$ of the AF layer, in consideration of the competition of the RKKY coupling with the AF coupling rather than with the direct coupling of the FM layer in the pinholes.

In summary, only at temperatures close to the $T_{N}$, a significant oscillatory interlayer exchange bias field for the $\mathrm{NiO} / \mathrm{Cu} / \mathrm{NiFe}$ structure with the $\mathrm{Cu}$ layer was observed. The oscillation was strongly depressed when lowering the temperature. The significant $T$ dependence of the interlayer exchange coupling is believed to be due to the $T$-dependent competition between the RKKY-like coupling and the AF coupling within the AF layer as well as the interlayer dipolar interaction. Our findings indicate the important effect of the temperature, and clarify the contradictory previous reports on the interlayer exchange bias coupling.

This work was supported by National Science Council of Taiwan under Grant No. NSC 89-2119-M-002-014 and by the Topic Project of Academia Sinica.
*Corresponding author. Email address: mtlin@phys.ntu.edu.tw

${ }^{1}$ P. Grünberg, R. Schreiber, Y. Pang, M. B. Brodsky, and H. Sowers, Phys. Rev. Lett. 57, 2442 (1986).

${ }^{2}$ S. S. P. Parkin, R. Bhadra, and K. P. Roche, Phys. Rev. Lett. 66, 2152 (1991).

${ }^{3}$ P. Bruno and C. Chappert, Phys. Rev. Lett. 67, 1602 (1991).

${ }^{4}$ P. Bruno and C. Chappert, Phys. Rev. B 46, 261 (1992).

${ }^{5}$ A. P. Malozemoff, Phys. Rev. B 35, 3679 (1987).

${ }^{6}$ A. P. Malozemoff, Phys. Rev. B 37, 7673 (1988).

${ }^{7}$ N. C. Koon, Phys. Rev. Lett. 78, 4865 (1997).

${ }^{8}$ C. Mauri, H. C. Siegmann, P. S. Bagus, and E. Kay, J. Appl. Phys. 62, 3047 (1987).

${ }^{9}$ J. Nogués, D. Lederman, T. J. Moran, and I. K. Schuller, Phys. Rev. Lett. 76, 4624 (1996).

${ }^{10}$ J. Nogués, T. J. Moran, D. Lederman, I. K. Schuller, and K. V. Rao, Phys. Rev. B 59, 6984 (1999).

${ }^{11}$ C. Leighton, J. Nogués, B. J. Jösson-Åkerman, and I. K. Schuller, Phys. Rev. Lett. 84, 3466 (2000).

${ }^{12}$ N. J. Gökemeijer, T. Ambrose, and C. L. Chien, Phys. Rev. Lett. 79, 4270 (1997).

${ }^{13}$ L. Thomas, A. J. Kellock, and S. S. P. Parkin, J. Appl. Phys. 87, 5061 (2000).

${ }^{14}$ T. Mewes, B. F. P. Roos, S. O. Demokritov, and B. Hillebrands, J. Appl. Phys. 87, 5064 (2000).

${ }^{15}$ T. Ambrose and C. L. Chien, Phys. Rev. Lett. 76, 1743 (1996).
${ }^{16}$ J. J. d. Miguel, A. Cebollada, J. M. Gallego, R. Miranda, C. M. Schneider, P. Schuster, and J. Kirschner, J. Magn. Magn. Mater. 93, 1 (1991).

${ }^{17}$ F. Petroff, A. Barthelemy, D. H. Mosca, D. K. Lottis, A. Fert, P. A. Schroeder, W. P. Pratt, R. Loloee, and S. Lequien, Phys. Rev. B 44, 5355 (1991).

${ }^{18}$ S. S. P. Parkin, Z. G. Li, and D. J. Smith, Appl. Phys. Lett. 58, 2710 (1991).

${ }^{19}$ P. Miltényi, M. Gierlings, J. Keller, B. Beschoten, G. Güntherodt, U. Nowak, and K. D. Usadel, Phys. Rev. Lett. 84, 4224 (2000).

${ }^{20}$ S. Demokritov, E. Tsymbal, P. Grünberg, W. Zinn, and I. K. Schuller, Phys. Rev. B 49, 720 (1993).

${ }^{21}$ D. Altbir, M. Kiwi, R. Ramirez, and I. K. Schuller, J. Magn. Magn. Mater. 149, L246 (1995).

${ }^{22}$ D. Altbir, J. d. A. e. Castro, and P. Vargas, Phys. Rev. B 54, R6823 (1996).

${ }^{23}$ J. S. Yang, J. Lee, and C.-R. Chang, IEEE Trans. Magn. 34, 2469 (1998).

${ }^{24}$ Ultrathin Magnetic Structures, edited by B. Heinrich and J. A. C. Bland (Springer-Verlag, Berlin, 1994), Vol. II.

${ }^{25}$ J. R. Cullen and K. B. Hathaway, Phys. Rev. B 47, 14998 (1993).

${ }^{26}$ Z. Q. Qiu, J. E. Mattson, C. H. Sowers, U. Welp, S. D. Bader, H. Tang, and J. C. Walker, Phys. Rev. B 45, 2252 (1992).

${ }^{27}$ D. M. Edwards, J. Mathon, R. B. Muniz, and M. S. Phan, J. Phys.: Condens. Matter 3, 4941 (1991). 